\title{
Recovery of Rare-Earth Elements from Neodymium Magnet Waste Using Glycolic, Maleic, and Ascorbic Acids Followed by Solvent Extraction
}

\author{
Marino Gergoric $^{1}$ (D) $\cdot$ Antonin Barrier ${ }^{2} \cdot$ Teodora Retegan $^{1}$
}

Published online: 12 December 2018

(c) The Author(s) 2018

\begin{abstract}
Rare-earth elements (REEs) play a key role in modern societies as their usage keeps increasing in new technologies and green energy sources. However, they are also considered the most critical raw materials in the EU and the USA in terms of supply. There is an increased global interest in the recycling of REEs from end-of-life products and industrial waste. Some REEs, such as Nd, Pr, and Dy, can be recovered from neodymium magnets. These magnets are of special interest since they are present in various technological wastes, such as hard disk drives, electric generators for wind turbines, electric motors, etc. Separation of REEs from other magnet components, such as Fe, which is the main part of the alloy, and further reprocessing of REEs, is the main goal of this work. In this work, neodymium magnet powder was successfully leached using the fully combustible organic lixiviants maleic, glycolic, and ascorbic acids, in order to potentially decrease the usage of strong mineral acids in the hydrometallurgical recovery of REEs. Subsequently, the REEs were selectively extracted from these leachates. For this separation step, several phosphate extractants (TBP, D2EHPA, Cyanex 272, and 923) were investigated, alongside TODGA, which follows the CHON principle and is fully combustible, with no ash or acidic gases being produced. The influences of various diluents (1-octanol, cyclohexanone, hexane, pentane, and dodecane) on the extraction were also studied since the diluents can play an important role in the extraction process and increase selectivity between the extraction of REEs and other impurities. Leaching was shown to be more efficient with maleic and glycolic acids than with ascorbic acid, even at room temperature. Values above $95 \%$ were reached for REEs with $1 \mathrm{M}$ concentration and 1/80 solid/liquid ratio. For ascorbic acid, heating the leaching system to $70{ }^{\circ} \mathrm{C}$ allowed similar values to be reached. D2EHPA has shown good extraction properties for the recovery of REEs from both glycolic and maleic leachate, mostly with nonpolar diluents such as pentane and hexane, without extracting transition metals (distribution ratios under 0.1). TODGA also showed good extraction of REEs and selectivity between elements, but only in the maleic leachate. As expected, the increasing concentration of the D2EHPA led to the increased distribution ratios. Thus, glycolic, maleic, and ascorbic organic acids, which have not been used before for leaching of neodymium magnet waste, showed good potential for the recovery of REEs from neodymium magnets and for the further development of large-scale recovery processes for REEs.
\end{abstract}

Keywords Leaching $\cdot$ Solvent extraction $\cdot$ Organic acids $\cdot$ Neodymium magnets $\cdot$ Sustainability

\section{Introduction}

The contributing editor for this article was Brajendra Mishra.

Marino Gergoric

marino@chalmers.se

1 Nuclear Chemistry and Industrial Materials Recycling, Chemistry and Chemical Engineering, Chalmers University of Technology, 41296 Göteborg, Sweden

2 National School of Chemistry Montpellier, 34090 Montpellier, France
Rare-earth elements (REEs) are metallic elements that are used in a vast range of technological applications, especially in domains linked to green energy [1]. Their properties make some of them essential for wind turbines, electric car batteries, computer hard disk drives and even phosphorous lamps. Due to their supply risk, they have been categorized as the most critical elements in the EU [2]. With China providing almost $90-95 \%$ of the world's demand, exports can tighten according to domestic demand and political interest. 
Since their procurement is essential for a various range of applications, instead of simply trying to find new stocks, many studies have proposed ways to efficiently recycle the REE wastes produced by industry [3, 4] or recover REEs from domestic wastes. One such industrial waste is neodymium $(\mathrm{NdFeB})$ magnets. Their demand is increasing rapidly, especially due to the development of hybrid/electric cars, which need specific rechargeable batteries, and wind turbines [3]. The magnets are composed mainly of $\mathrm{Fe}, \mathrm{Nd}$, and $\mathrm{B}$, but up to four other REEs can be found; Dy, Pr, Gd, and $\mathrm{Tb}$. For instance, Dy is added to improve high-temperature performances and intrinsic coercivity [5]. The amount of neodymium magnet waste is expected to grow in the future, and it is seen as a feasible waste stream for the recovery of REEs. This would not entirely replace mining, but it would help stabilize the markets and lower the prices for countries that can only rely on recycling of industrial technological wastes [5]. The recycling of REEs is currently far from being feasible on an industrial scale and is still in its early stages. In 2011, despite research already containing numerous possible processes, only $1 \%$ of REEs end-of-life wastes was recycled using various existing techniques, such as gasphase extraction, pyrometallurgical, or hydrometallurgical methods [3]. As of today, only 7\% of the light REEs and 6\% of the heavy REEs are produced by recycling from secondary sources to meet the EU demand for REEs [6].

Hydrometallurgical methods of recycling were developed because of their low energy consumption in contrast to pyrometallurgical methods, where high temperatures are needed to melt the alloys. However, hydrometallurgical methods can also have some drawbacks, such as large waste generation and usage of nonenvironmentally friendly chemicals. The methods provide excellent extraction properties and good separation factors between the REEs and other impurities, if the correct chemicals are selected [3,7]. Numerous studies have been performed to determine the ideal reagents and experimental conditions for the treatment of most REEs [3, $5,7]$. Hydrometallurgical processes normally consist of two steps: dissolution of the material/waste and separation of the elements using solvent extraction or ion-exchange. Dissolution is usually performed by leaching the metals using acids of varying concentrations. The acids most used and developed for magnet recycling are $\mathrm{HCl}, \mathrm{H}_{2} \mathrm{SO}_{4}$, and $\mathrm{HNO}_{3}$ [8-12]. These have been examined for decades, meaning

Fig. 1 Structural formulas of glycolic acid, maleic acid, and ascorbic acid, respectively

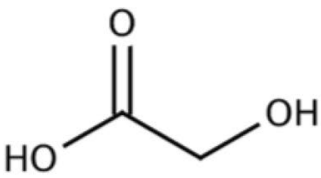

(a) the criteria affecting the efficiency of the leaching, such as acid concentration, solid/liquid (S/L) ratio, temperature, or even the use of the ultrasonic bath, have been studied in detail [13]. Despite their excellent leaching properties, using strong inorganic acids can have some drawbacks, such as poisonous gas evolution during leaching, adverse impact on the environment, soil acidification (in cases of release), and challenges during handling. Leaching has already been studied using some organic acids, such as acetic acid [14]. Acetic acid has the advantage of being a green solvent, it is easily degradable via aerobic and anaerobic conditions, and has possible regeneration capacities [15]. The literature on leaching of neodymium magnets using organic acids is, however, very scarce and should be studied further in order to determine their potential use for industrial-scale REEs reprocessing.

In this work, leaching of neodymium magnet waste powder was performed using maleic, glycolic, and L-ascorbic acids. These acids share several properties that make them interesting for a leaching process at industrial scale. They are mostly harmless (ascorbic acid being vitamin $\mathrm{C}$, and glycolic acid being used in skin care products), and become corrosive only at low $\mathrm{pH}$. They can also be quite cheap, thanks to some industrial processes using microbiological fermentation [16], and are easily disposable, being only composed of $\mathrm{C}, \mathrm{H}$ and $\mathrm{O}$ atoms. According to the $\mathrm{CHON}$ principle [3], they can be incinerated, releasing only $\mathrm{CO}_{2}$ and $\mathrm{H}_{2} \mathrm{O}$, in contrast to mineral acids (such as sulfuric acid or hydrochloric acid) that release toxic gases and need special handling precautions. Regarding their acidities, they can be ranked according to their first acidity $\mathrm{pKa}_{1}$ : maleic $(1.9)<$ glycolic $(3.8)<$ ascorbic (4.1) (meaning that maleic acid is the strongest of all three). Furthermore, it can be noted that both maleic and ascorbic acids are diacids $\left(\mathrm{pKa}_{2}: 6.1\right.$ and 11.7, respectively). Maleic is a strong diacid because of the double bond. Since this is the cis-isomer, it does not benefit from intramolecular hydrogen bonding and reacts more than the trans-isomer, fumaric acid. Glycolic acid is stronger than acetic acid thanks to the electron-withdrawing power of the hydroxyl group [17]. Finally, ascorbic acid is much more acidic than would be expected, thanks to its double bond that allows for stabilization by delocalization (Fig. 1). This could then beneficially improve the efficiency in the leaching of the neodymium magnet waste.

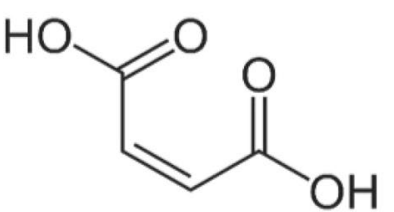

(b)<smiles>O=C1O[C@H]([C@@H](O)CO)[C@H](O)C1O</smiles>

(c) 
The following equations illustrate how the acids, represented as dissociated hydrogen cations, leach the rare-earth elements, regardless of their potential oxidized form [18]:

$$
\begin{aligned}
& 2 \mathrm{REE}+6 \mathrm{H}^{+} \rightarrow 2 \mathrm{REE}^{3+}+6 \mathrm{H}_{2} \\
& \mathrm{REE}_{2} \mathrm{O}_{3}+6 \mathrm{H}^{+} \rightarrow 2 \mathrm{REE}^{3+}+3 \mathrm{H}_{2} \mathrm{O} \\
& \mathrm{REE}(\mathrm{OH})_{3}+3 \mathrm{H}^{+} \rightarrow \mathrm{REE}^{3+}+3 \mathrm{H}_{2} \mathrm{O}
\end{aligned}
$$

The following equations illustrate the behavior of glycolate and maleate anions in solution, respectively, and their interactions with rare-earth cations, such as $\mathrm{Nd}$.

$$
\mathrm{Nd}^{3+}+j \mathrm{HOCH}_{3} \mathrm{COO}^{-} \leftrightarrows \mathrm{Nd}\left(\mathrm{OOCCH}_{3} \mathrm{OH}\right)_{j}^{3-j} \quad j=1,2,3
$$

$\mathrm{Nd}^{3+}+j \mathrm{HOOCC}_{2} \mathrm{H}_{2} \mathrm{COO}^{-} \leftrightarrows \mathrm{Nd}\left(\mathrm{OOCC}_{2} \mathrm{H}_{2} \mathrm{COOH}\right)_{\mathrm{j}}^{3-\mathrm{j}} \quad j=1,2,3$

If a solvent extraction step is performed afterward, several extracting agents have been developed industrially, i.e. organic phosphorous extractants, such as phosphoric acids. Cyanex 923 and D2EHPA (di-(2-ethylhexyl)phosphoric acid) are two examples [19, 20], but they sometimes suffer from various drawbacks, such as poor selectivity, poor stripping of the metals from the organic phase, and a low rate of extraction. Due to the need to develop greener and totally combustible products for the process, it would be beneficial to use extractants that follow the CHON principle, such as amides or carboxylic acids, such as tetraoctyl diglycolamide (TODGA) [10]. This specific extractant has shown great specificity for lanthanides and other bloc-f elements, extracting only divalent and trivalent ions with large ionic radii over $80 \mathrm{pm}$ [10]. Furthermore, the diluent in which the extracting agent is dissolved to form the organic phase (solvent) has great impact on the efficiency and selectivity of the extraction [10, 21-23]. Other criteria that affect the extraction process are also of great importance, such as acidic concentration in the aqueous phase, as this provides counter ions for the exchange mechanisms and complex formations.

For this work, several extractants and diluents were tested. To ascertain the extraction feasibility on an industrial scale, the following common phosphate extractants were tested: TBP (tributyl phosphate), D2EHPA, Cyanex 923 (mixture of trialkyl-phosphine oxides), and Cyanex 272 (di(2,4,4-trimethylpentyl) phosphinic acid), as well as TODGA (to determine whether a greener procedure could be feasible). Several diluents, such as alcohols (1-octanol), ketones (cyclohexanone), and alkanes (pentane, hexane, dodecane, and solvent 70), were also tested. The first goal was to achieve separation of the REEs from other elements, then to ascertain if they could be separated from each other, and finally to explore the potential efficiency of greener lixiviants for the leaching steps of magnet recycling for REE separation using the hydrometallurgical route. Due to their mostly harmless properties, these lixiviants could replace mineral acids and are much easier to recycle. If the leaching process is effective, some conditions would be mitigated, such as temperature or solid-to-liquid ratios, which were kept constant in the first tests. The leachates could then be used to test different extraction procedures, with different extractants and diluents, to ascertain the feasibility of a separation step with these specific leachates.

\section{Theory}

D2EHPA is an acidic extractant (extraction occurring according to the ion-exchange mechanism in Eq. 6), whereas TBP and TODGA are solvating extractants (the solvating mechanism is shown in Eq. 7), thus having a specific mechanism leading to different efficiencies. The following equations show the mechanisms of acidic extraction (6) and solvating extraction (7):

$\mathrm{Ln}^{3+}+\overline{x(\mathrm{HR})_{2}} \leftrightarrows \overline{\mathrm{LnR}_{3}(\mathrm{HR})_{2 x-3}}+3 \mathrm{H}^{+}$

$\mathrm{Ln}^{3+}+3 \mathrm{RCOO}^{-}+3 \overline{\mathrm{TBP}} \leftrightarrows \overline{\mathrm{Ln}(\mathrm{TBP})_{3}\left(\mathrm{RCOO}^{-}\right)_{3}}$

where $\mathrm{Ln}^{3+}$ represents the REE ion in solution; HR represents the acidic extractant molecules, for instance, D2EHPA (the bar meaning in organic solution); $\mathrm{RCOO}^{-}$represents the glycolate or maleate anions in aqueous solution; and TBP is the solvating extractant.

The efficiency of each extraction step is determined using the distribution ratio $(D)$ as the most important parameter, and separation factors $(\alpha)$. These are calculated using the following Eqs. (8) and (9), respectively:

$$
D_{\mathrm{A}}=\frac{[A]_{\mathrm{org}}}{[A]_{\mathrm{aq}}}
$$

$\alpha_{\overline{\mathrm{B}}}=\frac{D_{\mathrm{A}}}{D_{\mathrm{B}}}$,

where $[A]_{\mathrm{org}}$ and $[A]_{\mathrm{aq}}$ are the equilibrium concentrations of the metal of interest in all its existing species in the organic and aqueous phases, respectively. The separation factor represents the selectivity between two metals in the extraction.

\section{Experimental Procedure}

\section{Material Characterization}

Fine NdFeB magnet powder was procured for the experiments from the University of Birmingham [24]. Pretreatment heating was carried out by roasting the powder at $400{ }^{\circ} \mathrm{C}$ 
for $1.5 \mathrm{~h}$ in an Entech muffle furnace (LF2) in a ceramic crucible. After roasting, the powder was sieved to a particle size of $<355 \mu \mathrm{m}$ using a Retsch AS 200 vibratory sieve shaker. To determine the chemical composition of the magnet powder, $0.5 \mathrm{~g}$ of powder was dissolved in $20 \mathrm{~mL}$ of aqua regia at $70 \pm 1{ }^{\circ} \mathrm{C}$, heated for one hour on a heating plate, and the samples were analyzed after total dissolution with ICP-OES (ppm scale) to determine the weight quantities of $\mathrm{Nd}, \mathrm{Pr}, \mathrm{Dy}, \mathrm{Fe}, \mathrm{Co}$, and B in the magnet. Experiments were done in triplicates.

Aqua regia was prepared by mixing concentrated $\mathrm{HNO}_{3}$ $\left(65 \%\right.$, suprapur ${ }^{\odot}$, Merck) and hydrochloric acids (37\%, ACS reagent, Sigma-Aldrich), in 1:3 volume ratio, respectively. Samples were diluted with $1 \mathrm{M} \mathrm{HNO}_{3}$ before the ICP-OES measurement. The same method was used throughout the experiments, with a calibration curve prepared using standard solutions.

\section{Leaching Experiments}

Leaching experiments were carried out using glycolic acid (Sigma-Aldrich, $>99.5 \%$ ), maleic acid (Sigma-Aldrich, $>99.5 \%$ ), and L-ascorbic acid (Sigma-Aldrich, > 99.5\%), in $50 \mathrm{~mL}$ polypropylene bottles, with constant agitation using magnetic stirrers. Leaching agents were prepared by diluting the concentrated acids with MilliQ water (Merck Millipore Q-POD ${ }^{\odot}$ ). The volume of the different acids remained constant, $25 \mathrm{~mL}$, and changes were made to: (1) acid concentration, (2) S/L ratios, (3) temperature, and (4) stirring speed. The influence of acid concentration was investigated for each acid with the S/L ratio fixed at $1 / 50 \mathrm{~g} / \mathrm{mL}$, temperature set at $25 \pm 1{ }^{\circ} \mathrm{C}$ and concentration ranging from 0.6 to $1 \mathrm{M}$. S/L ratio was then varied from $1 / 30$ to $1 / 80 \mathrm{~g} / \mathrm{mL}$ using $1 \mathrm{M}$ of each acid. Furthermore, the temperature was varied between $25 \pm 1{ }^{\circ} \mathrm{C}$ and $70 \pm 1{ }^{\circ} \mathrm{C}$. Finally, rotation speed was varied between 400 and $1000 \mathrm{rpm}$.

For the kinetic studies, $0.1 \mathrm{~mL}$ samples were collected after 100, 200, 300, $400 \mathrm{~min}$, and after $24 \mathrm{~h}$, with the agitation stopped so that particles could deposit. The samples were diluted with $1 \mathrm{M} \mathrm{HNO}_{3}$ and analyzed by ICP-OES. Experiments were conducted in triplicate to ascertain reproducibility. The heating and stirring plate used was an IKA ${ }^{\oplus}$ RT15. Powder was weighed on a Fisher MH-214 analytical scale. The $\mathrm{pH}$ values of the acids before and after leaching were measured using a MeterLab ${ }^{\mathrm{TM}}$ PHM $240 \mathrm{pH} /$ ion Meter $\mathrm{pH}$ electrode.

\section{Liquid-Liquid Extraction Experiments}

Five organic extractants were investigated: TBP (SigmaAldrich, 97\%), D2EHPA (Sigma-Aldrich, 97\%), TODGA, Cyanex 272 (Cytec, 85\%), and Cyanex 923 (Cytec, 93\%). TODGA was synthesized at the Nuclear Chemistry

Department of Chalmers Institute of Technology and 98\% purity was ascertained by NMR. Solutions of $1 \mathrm{M}$ concentration were prepared by dilution, using $6 \mathrm{~mL}$ for the TODGA and $20 \mathrm{~mL}$ for all others. For other experiments, 0.2, 0.4, 0.6 , and $0.8 \mathrm{M}$ solutions of the best extractant were then prepared to determine the influence of concentration in the organic phase on extraction efficiency. The first diluent chosen was Solvent 70 (hydrocarbons C11-C14, < aromatics, Statoil, Sweden). Furthermore, the influence of the diluent was tested using 1-octanol (Sigma-Aldrich, Cylcohexanone (>99\%, ACS reagent, Sigma-Aldrich), pentane (SigmaAldrich, Hexane (95\%, anhydrous, Sigma-Aldrich) and n-dodecane (99\%, Sigma-Aldrich) and compared to Solvent 70. Leachates were prepared using $100 \mathrm{~mL}$ of glycolic acid and $100 \mathrm{~mL}$ of maleic acid, $1 / 80 \mathrm{~S} / \mathrm{L}$ ratio, at room temperature and with a stirring speed of $400 \mathrm{rpm}$, for $400 \mathrm{~min}$. Extractions were performed by putting $1 \mathrm{~mL}$ of aqueous leachate in contact with $1 \mathrm{~mL}$ of organic phase. The shaking vials were shaken for $10 \mathrm{~min}$ at $25 \pm 1^{\circ} \mathrm{C}$, using an IKA VIBRAX VXR Basic at 1500 vibrations per minute, and then centrifuged for $1 \mathrm{~min}$ at $5000 \mathrm{rpm}$ in a Heraeus Labofuge 200 centrifuge. Samples of the remnant aqueous phase were collected for ICP-OES analysis.

\section{Results and Discussion}

\section{Magnet Powder Characterization}

To quantify the elemental composition of the magnet powder, leached samples after aqua regia treatment were diluted in $1 \mathrm{M} \mathrm{HNO}_{3}$ and analyzed by ICP-OES to determine the amount of Nd, Pr, and Dy as REEs, as well as Fe, Co, and $\mathrm{B}$ as other components. The main valuable REE here is $\mathrm{Nd}$ (Table 1), forming $20.4 \%$ of the magnet, and as expected the other main component of the alloy is Fe (52.7\%). Leaching kinetics will mostly focus on REEs, such as Nd, Pr, and Dy, as other elements are not the primary target of this research. The total sum of the percentages is $78.4 \%$, meaning that some oxides might have formed after roasting. Furthermore, small percentages of the magnet also occasionally contain $\mathrm{Ni}$ and $\mathrm{Al}$, but these were not monitored in detail in this research.

Table 1 Composition of the magnet powder leached with aqua regia. The leachate was diluted with $1 \mathrm{M} \mathrm{HNO}_{3}$ and measurement was performed with ICP-OES iCAP 6500, Thermo Fischer (ppm scale)

\begin{tabular}{ll}
\hline Element & Mass/\% \\
\hline $\mathrm{Nd}$ & $20.4 \pm 0.2$ \\
$\mathrm{Pr}$ & $2.36 \pm 0.01$ \\
$\mathrm{Dy}$ & $0.74 \pm 0.01$ \\
$\mathrm{Fe}$ & $52.7 \pm 0.7$ \\
$\mathrm{Co}$ & $1.18 \pm 0.01$ \\
$\mathrm{~B}$ & $0.94 \pm 0.01$ \\
\hline
\end{tabular}




\section{Leaching Kinetics and Concentration Effect}

Keeping temperature and solid/ratios equal $\left(25 \pm 1{ }^{\circ} \mathrm{C}\right.$ and $1 / 50 \mathrm{~g} / \mathrm{mL}$ ), magnet powder was dissolved in glycolic, maleic, and ascorbic acid solutions, at $0.6 \mathrm{M}, 0.8 \mathrm{M}$, and $1 \mathrm{M}$, respectively (Fig. 2). As expected, leaching efficiency increases with increasing acid concentration. For instance, $\mathrm{Nd}$ extraction increased from $78.4(0.6 \mathrm{M})$ to $86.7 \%(1 \mathrm{M})$ after $400 \mathrm{~min}$. After $400 \mathrm{~min}$, reactions with glycolic and maleic acids reached equilibrium, and concentrations remained high and globally unchanged. For ascorbic acid, however, the equilibrium was not reached within $24 \mathrm{~h}$ and concentrations remained significantly lower than for the other acids. Generally, $1 \mathrm{M}$ concentrations seem to be the most efficient and will be used in further studies. Maleic acid showed better leaching properties than glycolic acid, especially at 0.6 and $0.8 \mathrm{M}$. Ascorbic was the weakest of all 3 acids, therefore efficiency seems to correlate with the pKa ranking of the acids. Furthermore, $400 \mathrm{~min}$ is sufficient to reach equilibrium and for further experiments samples will only be collected at $400 \mathrm{~min}$. The influence of acid concentration can be explained by the drop in $\mathrm{pH}$ and rise of the acidity of the solution, allowing better conditions to dissolve REEs and other metals. All the elements analyzed were efficiently dissolved with maleic and glycolic acids (between 80 and $90 \%$ at $1 \mathrm{M}$ after $400 \mathrm{~min}$ ). This is good and equivalent to some inorganic acid leaching and will be improved by optimizing different conditions, such as temperature and S/L ratio. The $\mathrm{pH}$ values of the maleic acid (0.6-1 M) before leaching were around 1 , glycolic acid values $(0.6-1 \mathrm{M})$ were around 2 and the ascorbic values $(0.6-1 \mathrm{M})$ were around 2.2, with only slight variation of the $\mathrm{pH}$ value with the varying concentration. A rise in $\mathrm{pH}$ was noticed, with the values dropping to around 3.5, 4 and 4.5 in maleic, glycolic, and ascorbic acids, respectively. Slight variations were noticed between the different concentrations used. The $\mathrm{pH}$ increase could explain the slight loss of leaching efficiency of $\mathrm{Fe}$ and REEs in glycolic and maleic acids, since some precipitation or suspension of precipitate might have occurred in the system.

\section{Solid/Liquid Ratios}

Using data from previous experiments, the influence of $\mathrm{S} / \mathrm{L}$ ratios was tested, keeping $1 \mathrm{M}$ concentrations and $25 \pm 1{ }^{\circ} \mathrm{C}$ temperature. The ratios tested were $1 / 80,1 / 50$, and $1 / 30 \mathrm{~g} /$ $\mathrm{mL}$ (Fig. 3), keeping the same magnet mass but changing volume of acids $(15 \mathrm{~mL}$ and $40 \mathrm{~mL}$ for $1 / 30$ and $1 / 80 \mathrm{~g} / \mathrm{mL}$, respectively). It is theorized that smaller $\mathrm{S} / \mathrm{L}$ ratios are more efficient because there is more liquid (acid) available to dissolve the solid. This is proven by the results obtained, as $\mathrm{Nd}$ recovery increased from $41(1 / 30)$ to $50 \%(1 / 80)$ in ascorbic acid. Dissolution can be as high as $95 \%$ for the $\mathrm{S} / \mathrm{L}$ ratio of
$1 / 80 \mathrm{~g} / \mathrm{mL}$ in glycolic acid, which shows the importance of this criterion, allowing leaching with organic acids to reach completion and values usually obtained when leaching with inorganic acids.

\section{Temperature Effect}

After the S/L ratio, the effect of temperature was studied, first at room temperature $\left(25 \pm 1{ }^{\circ} \mathrm{C}\right)$, and then at $50 \pm$ $1{ }^{\circ} \mathrm{C}$ and $70 \pm 1{ }^{\circ} \mathrm{C}$ (Fig. 4). Acid concentration was kept at $1 \mathrm{M}$ and $\mathrm{S} / \mathrm{L}$ ratio at $1 / 80 \mathrm{~g} / \mathrm{mL}$, since these were the most efficient experimental conditions previously obtained. Heat is an important parameter, as high temperatures usually make species more soluble, as this provides energy to break the bonds (ionic, covalent) between molecules and atoms, leading to solvation of the formed ions. According to other researchers, metal leaching reactions are mostly endothermic and are favored by high temperatures that shift the equilibrium in favor of dissolution $[9,15]$. The issue is that all elements contained in neodymium magnets follow this very trend, so the dissolution rates of $\mathrm{Fe}$ and other unwanted elements also rise as temperature increases. For maleic acid, since the conditions already allowed $>90 \%$ leaching, few changes have been observed, even if most of the time a small increase in leaching efficiency was noticed. For ascorbic acid, however, the rise was clear and noticeable (from 50\% for neodymium at room temperature to $95 \%$ at $70 \pm 1{ }^{\circ} \mathrm{C}$ ), and this rise was observed for all elements. Temperature is the only condition that allowed ascorbic leaching to reach 90\% efficiency. For glycolic acid, however, an opposite trend occurred, as efficiency dropped clearly with temperature. This can be explained by the low boiling point [25] at which decomposition also occurs [26]. With this decomposition and volume loss, the strength of the solution diminished, and leaching was less efficient. For these reasons, further experiments will be performed at room temperature for leaching with glycolic and maleic acids, and it is advised to heat to $70{ }^{\circ} \mathrm{C}$ for ascorbic acid use.

\section{Stirring Speed}

Lastly, the rotation speed of the magnetic stirrer was investigated with $1 \mathrm{M}$ acids, $1 / 80 \mathrm{~g} / \mathrm{mL} \mathrm{S} / \mathrm{L}$ ratio, at $25 \pm 1{ }^{\circ} \mathrm{C}$. At low rotation speeds leaching can be restricted by matter transfer, so speeds were varied between 400, 700 and $1000 \mathrm{rpm}$ to see if such limitations could occur. If the stirring is too slow, magnets particles tend to aggregate on the stirrer, which diminishes the surface available for the acid to reach. The results show that $400 \mathrm{rpm}$ is sufficient for leaching to take place normally, as no significant changes were seen between samples. 
Fig. 2 Efficiencies of leaching with different acids (glycolic, maleic, and ascorbic) and acid concentrations $(0.6 \mathrm{M}, 0.8 \mathrm{M}$, and $1 \mathrm{M}$ ), after 100, 200, 300, $400 \mathrm{~min}$, and $24 \mathrm{~h}$, in percentages of total a Nd, b Pr, c Dy, and $\mathbf{d}$ Fe dissolved in the solution. Temperature was kept at $25 \pm 1{ }^{\circ} \mathrm{C}$, solid/liquid ratio was $1 / 50 \mathrm{~g} / \mathrm{mL}$, and stirring speed was $400 \mathrm{rpm}$ (Color figure online)
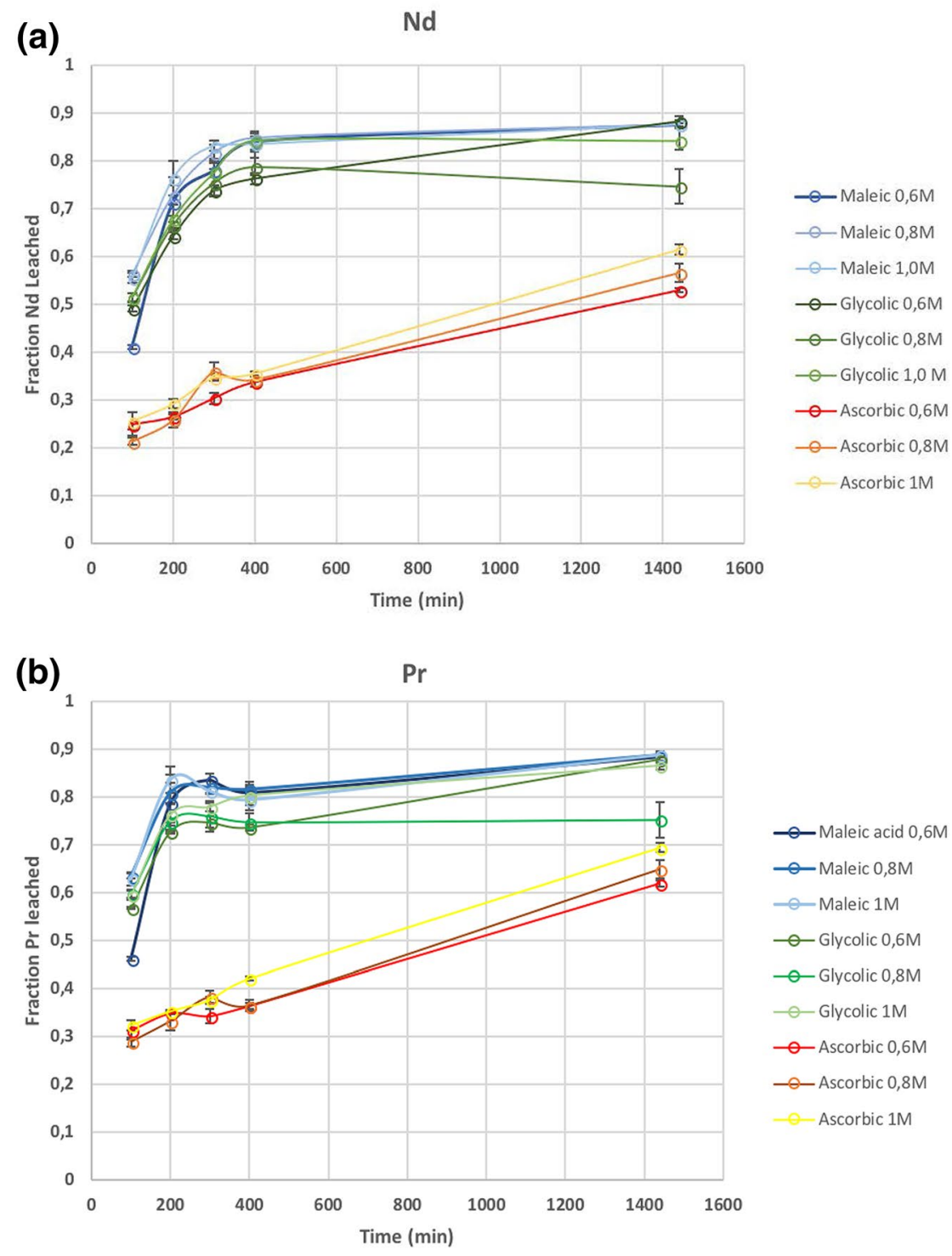

(c)

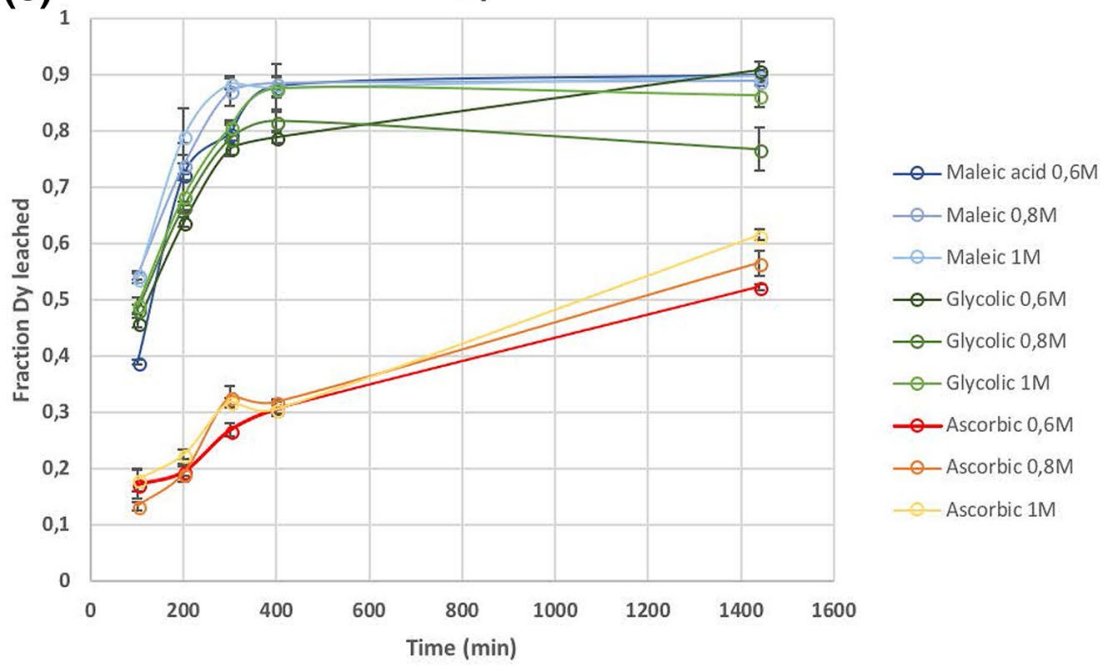


Fig. 2 (continued)

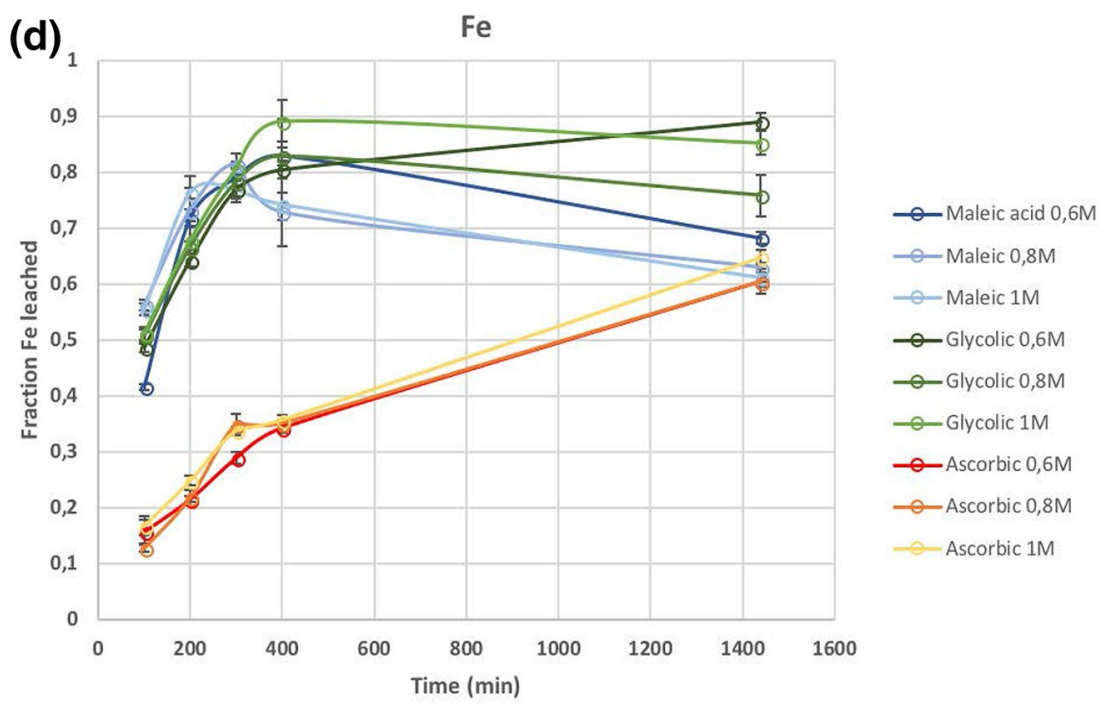

\section{Treatment of Leached Solutions}

At the end of the leaching experiments small amounts of solid were noticed floating in the solutions. Previous studies showed that these were mostly nickel and coating that remained undissolved regardless of the experimental conditions [10]. The leachates were filtered using syringes and $0.45-\mu \mathrm{m}$ propylene filters. Most solutions were transparent, especially for yellowish and orange maleic and glycolic acids. Ascorbic acid solutions were darker because of the oxidation of ascorbic acid. Some glycolic acid filtrate, after being left for more than 2 days, started to precipitate. Precipitation mostly occurred in weaker acidic conditions, for example, at $0.6 \mathrm{M}$ and at $1 / 30 \mathrm{~g} / \mathrm{mL} \mathrm{S} / \mathrm{L}$ ratio (pH between 4 and 4.5). These residues were filtered and dried. They were easily dissolved in $1 \mathrm{M} \mathrm{HCl}$ and analyzed by ICP-OES. The analysis of the composition showed that it contained relatively twice as much $\mathrm{Fe}$ as the magnet material, in comparison to other metals, such as $\mathrm{Nd}$. Co was also present in high quantities. The residues were further analyzed by XRD and SEM (Fig. 5). The microscopic structure of the precipitate can be observed in the SEM image. It consists mostly of large particles containing mainly $\mathrm{Fe}$ and $\mathrm{O}$ (point 1 on Fig. 5), while some areas contain large amounts of $\mathrm{Nd}$ (around $60 \%$ - point 2 on Fig. 5) and locally dispersed smaller shards (tubular particulates) of $\mathrm{Nd}$. Other elements were not detected as they were present in too small quantities. XRD analysis was inconclusive, due to the high amounts of different species and organic molecules. Traces of glycolates, such as neodymium glycolate, were found.

\section{Liquid-Liquid Extraction}

After the preliminary tests using liquid-liquid extraction (Fig. 6), it was concluded that D2EHPA was the best extractant for selective extraction of the REEs from glycolic and maleic acid leachates, while keeping other metals mostly in the aqueous phase. TODGA showed promising results in the maleic acid leachate. Other extractants tested all showed lower D values for REEs, so that the separation factor between them and transition metals was poor and not sufficient for an efficient separation step. These differences of extraction efficiency can be explained by the fact that the extractants tested had different mechanisms of extraction, for instance D2EHPA is an acidic extractant, whereas TBP, TODGA and Cyanex 923 are solvating extractants. This means that, according to Eq. (7), solvating extractants require anions, present in the aqueous phase, to form complexes with the extractant molecules and metallic cations. Thus, a change in the leachates means a change of the counter-ions in the solution, meaning the extraction mechanism will be compromised. This was seen with TODGA in the glycolic leachate. It seems, however, that extraction of REEs using TODGA was not hindered in the maleic acid leachate. For acidic extractants, Eq. (6) shows that cations are directly exchanged with the hydrogen atoms of the extractants, regardless of the counter-ions. From the results obtained, the following optimization tests were performed using D2EHPA as an extractant.

Some color changes were observed during the experiments. At first the aqueous phase was yellow, and this remained the same for some experiments (for instance with TODGA), whereas in other experiments, such as Cyanex 923 with maleic leachate, the inverse was seen, and the organic phase became yellow. For D2EHPA, both phases became transparent. In the case of Cyanex 923 with glycolic leachate, a third phase was observed, appearing to be an emulsion and insoluble in both aqueous and organic media. This phenomenon can also appear during industrial processes, such as the PUREX process, and should be avoided [27]. 


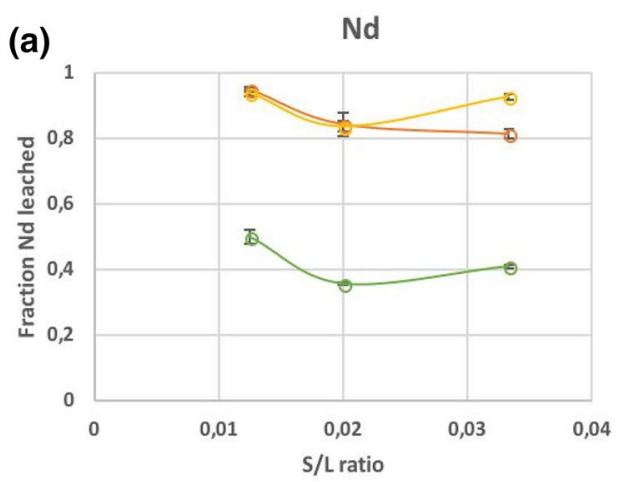

- - Glycolic acid
- Maleic acid
$\odot$ - Ascorbic acid
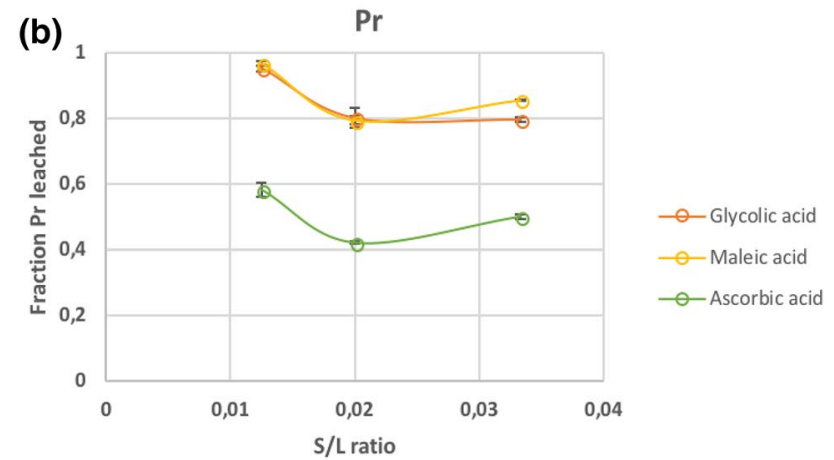

(c)
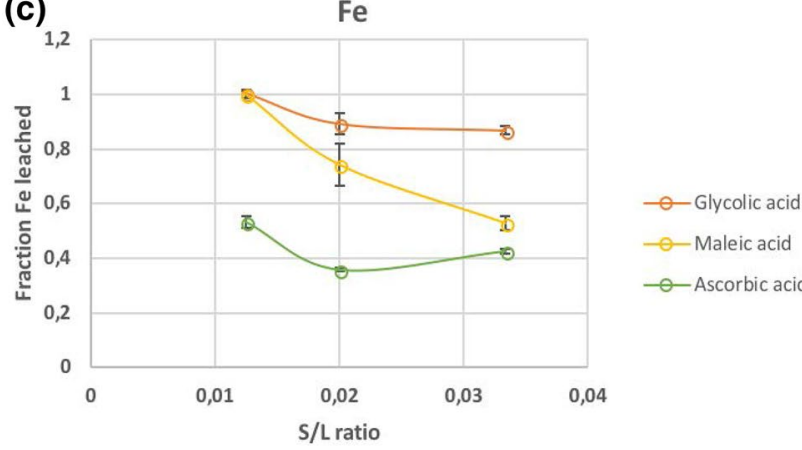

Fig. 3 Efficiencies of leaching with different acids (glycolic, maleic, and ascorbic) at different solid/liquid ratios (1/80, 1/50, and 1/30 g/ $\mathrm{mL}$ ), after $400 \mathrm{~min}$, shown as percentages of total a $\mathrm{Nd}$, b Pr, c Dy, and $\mathbf{d}$ Fe dissolved in the solution. Temperature was kept at $25 \pm$ $1{ }^{\circ} \mathrm{C}$, concentration was $1 \mathrm{M}$, and stirring speed was $400 \mathrm{rpm}$ (Color figure online)

Since D2EHPA proved to be the best extractant for both leachates, it was used to perform experiments to determine the influence of concentration. Solutions of D2EHPA in Solvent 70 were prepared, at $0.2,0.4,0.6,0.8$, and $1 \mathrm{M}$. It was expected that the extractions efficiencies would increase with the concentration, as there are more extractant molecules available to reach and solvate metal ions. This was confirmed by this study (Fig. 7), as 1 M D2EHPA proved to be the most efficient of the five concentrations tested. For neodymium the rise was almost linear, whereas for other elements there were more variations. However, if a concentration augmentation is preferable for REEs, this also increases
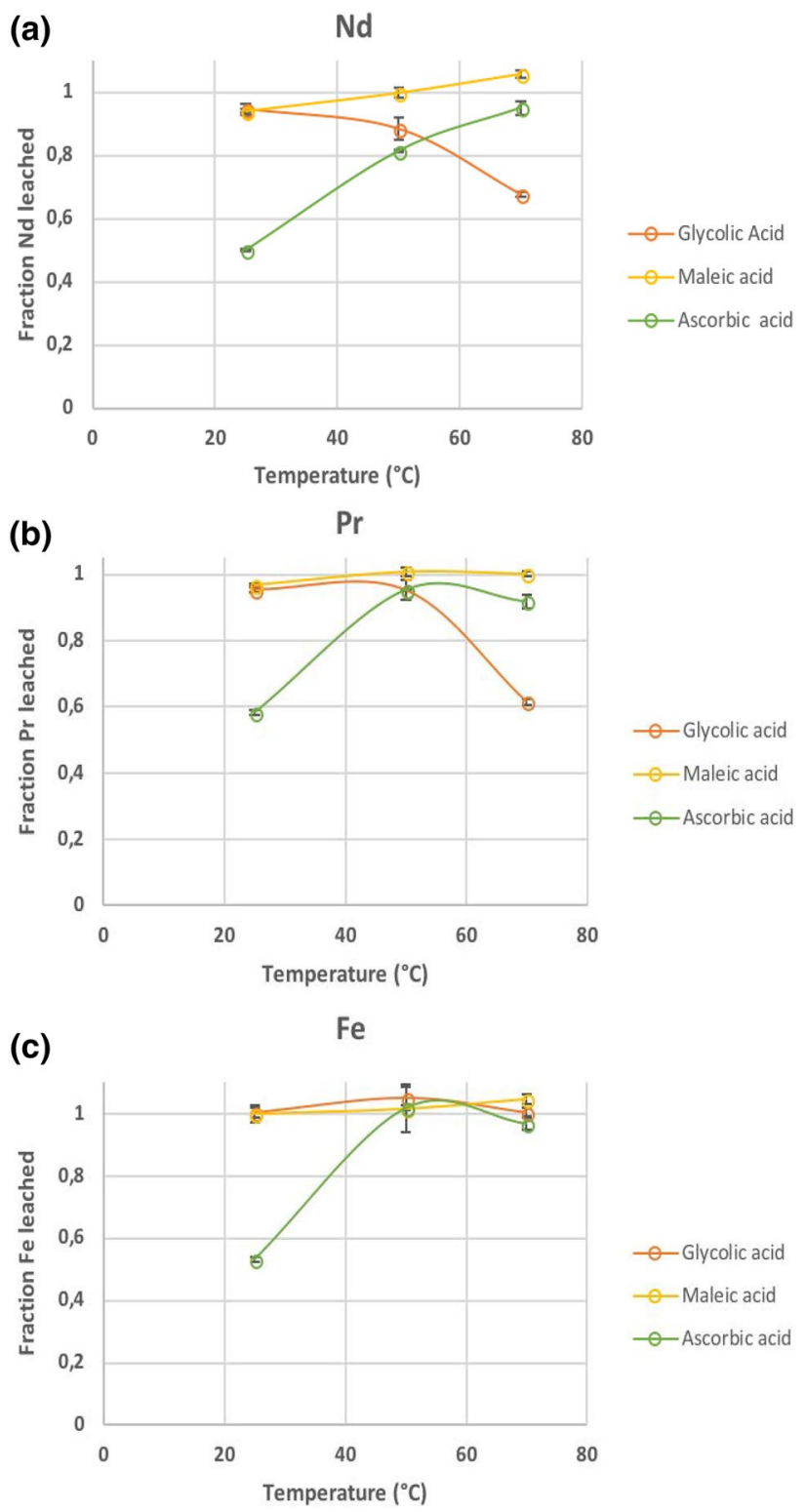

Fig. 4 Efficiency of leaching with different temperatures $(25,50$ and $75^{\circ} \mathrm{C}$ ), shown as percentage of total $\mathbf{a ~} \mathrm{Nd}, \mathbf{b} \mathrm{Pr}$, and $\mathbf{c}$ Fe leached. The solid/liquid ratio was kept at $1 / 80 \mathrm{~g} / \mathrm{mL}$, the stirring speed was $400 \mathrm{rpm}$, and samples were collected after $400 \mathrm{~min}$ (Color figure online)

the D values of transition metals. Thus, for a better separation, the concentration of the D2EHPA can be altered, as well as the $\mathrm{pH}$ of the leachate. For subsequent tests, however, the $1 \mathrm{M}$ concentration will be chosen to encompass all the elements and the effect of diluents on their extraction.

\section{Diluent Effect on Solvent Extraction}

Lastly, the influence of the diluent used for the organic phase was tested (Fig. 8), as differences between the 


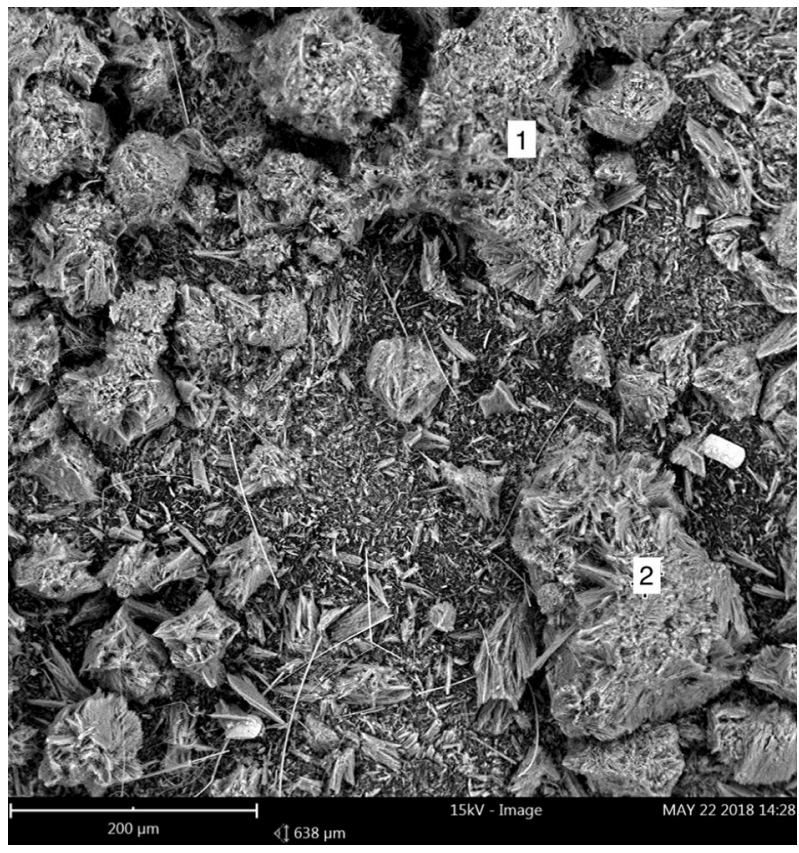

Fig. 5 SEM magnification $(\times 4100,10 \mathrm{kV}-$ Map) of the filtered residues after leaching of the roasted $\mathrm{NdFeB}$ powder with $0.6 \mathrm{M}$ glycolic acid for over $24 \mathrm{~h}$. The spots 1 and 2 in the image show the locations where the EDS analysis was performed

\section{(a) Extraction from glycolic leachate}

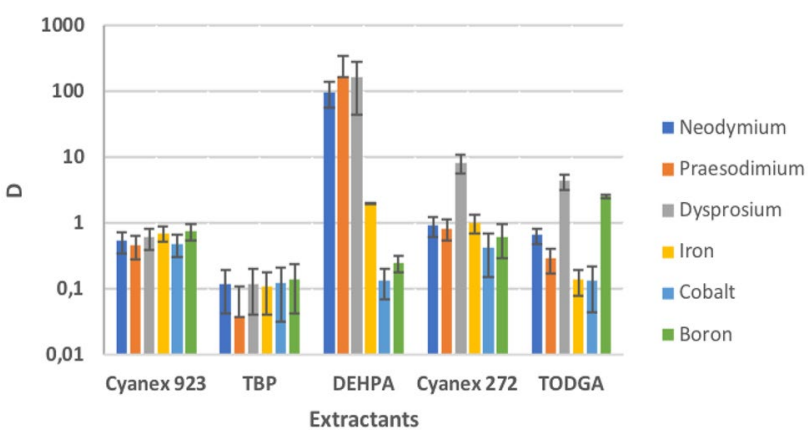

(b) Extraction from maleic leachate

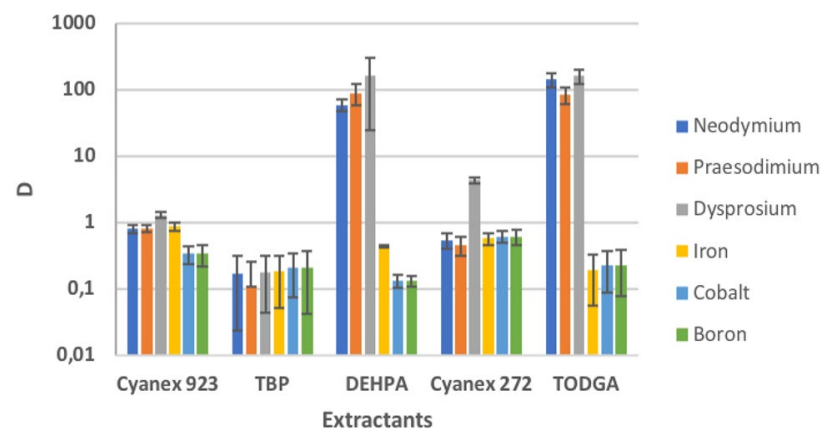

Fig. 6 The dependence of distribution ratios for Nd, Pr, Dy, Fe, Co, and $\mathrm{B}$ in $1 \mathrm{M}$ solutions of different extractants (Cyanex 272, 923, TBP, D2EHPA, and TODGA) in Solvent 70. The aqueous phase was $1 \mathrm{M}$ $\mathbf{a}$ glycolic acid and $\mathbf{b}$ maleic acid; the organic-to-aqueous phase ratio was $1: 1$, and the temperature was $25 \pm 1^{\circ} \mathrm{C}$ (Color figure online)

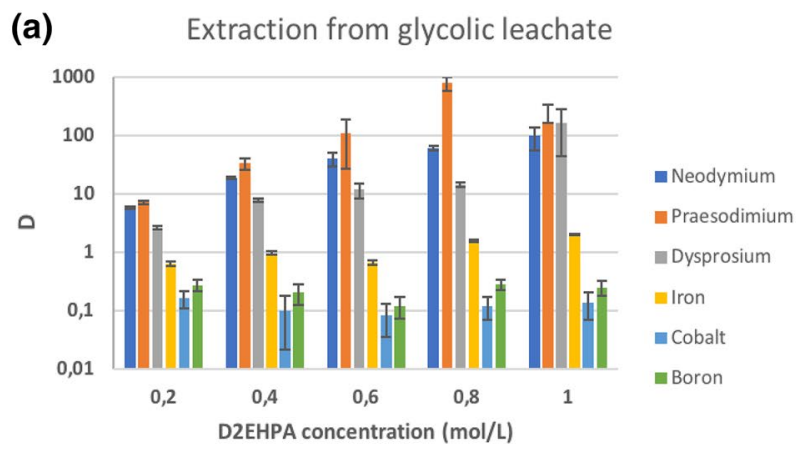

(b) Extraction from maleic leachate
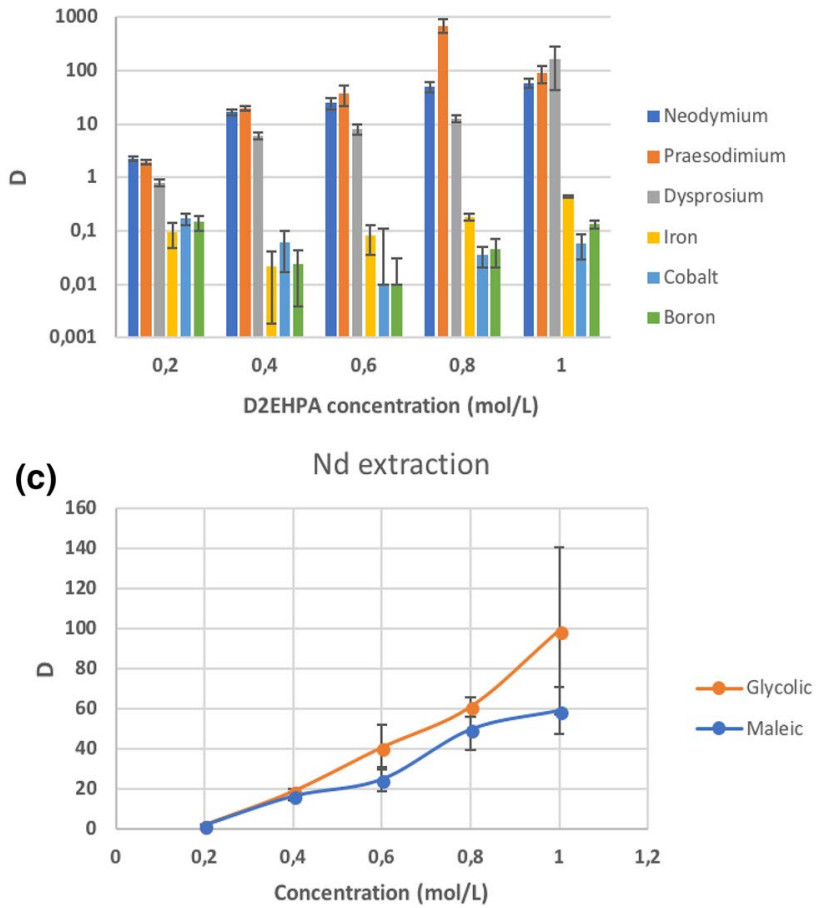

Fig. 7 The dependences on distribution ratios of Nd, Pr, Dy, Fe, Co, and $\mathrm{B}$ after extraction in solutions of D2EHPA in Solvent 70, at different concentrations $(0.2,0.4,0.6,0.8$, and $1 \mathrm{M})$. The aqueous phase was $1 \mathrm{M}$ a glycolic acid and $\mathbf{b}$ maleic acid; organic-to-aqueous phase ratio was $1: 1$ and the temperature was $25 \pm 1{ }^{\circ} \mathrm{C}$ (Color figure online)

chemical and physical properties of the diluent can affect the extraction mechanisms. It is mostly the polarity and the ability to form hydrogen bonds and covalent bonds that matters when choosing a diluent. Previous studies have shown that nonpolar diluents, such as Solvent 70 , were best for an extraction process using hydrophobic extractants, such as TODGA with its long alkyl chains [10]. Experimental results obtained in this research showed that D2EHPA extraction efficiency decreases in the pentane $>$ hexane $>$ Solvent $70>$ dodecane sequence. Octanol and cyclohexanone were deemed unsuitable as diluents as they extracted larger amounts of cobalt and boron, and fewer quantities of REEs than nonpolar solvents. 
Fig. 8 The dependences on distribution ratios of $\mathrm{Nd}, \mathrm{Pr}$, $\mathrm{Fe}$, and $\mathrm{Co}$ in $1 \mathrm{M}$ solutions of D2EHPA in different diluents (1-octanol, cyclohexanone, pentane, hexane, dodecane, and Solvent 70). The aqueous phase was $1 \mathrm{M}$ glycolic or maleic aci;, the organic-to-aqueous phase ratio was $1: 1$ and the temperature was $25 \pm 1{ }^{\circ} \mathrm{C}$ (Color figure online) (a)

$\mathrm{Nd}$

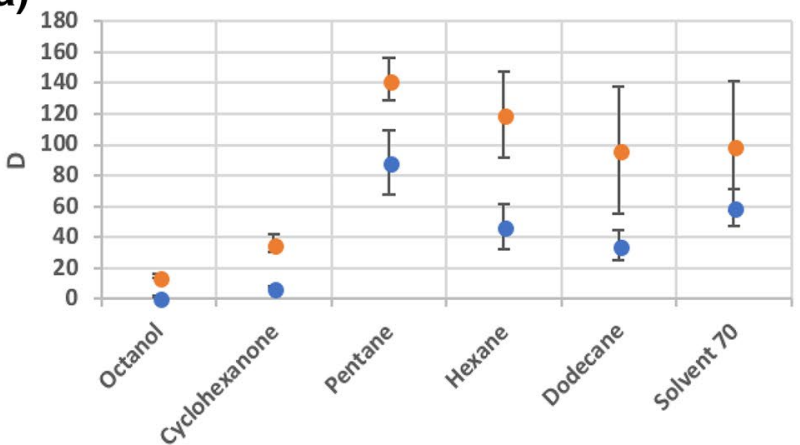

- Glycolic

- Maleic

Diluents

(b)

$\mathrm{Pr}$

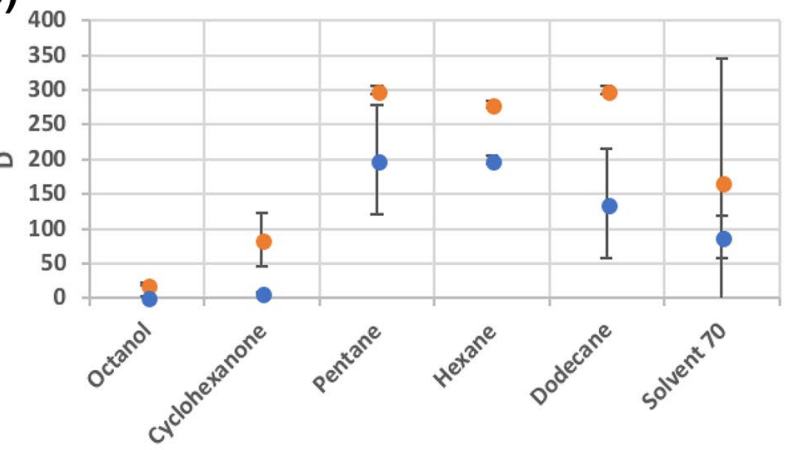

Diluents

(c)

$\mathrm{Fe}$

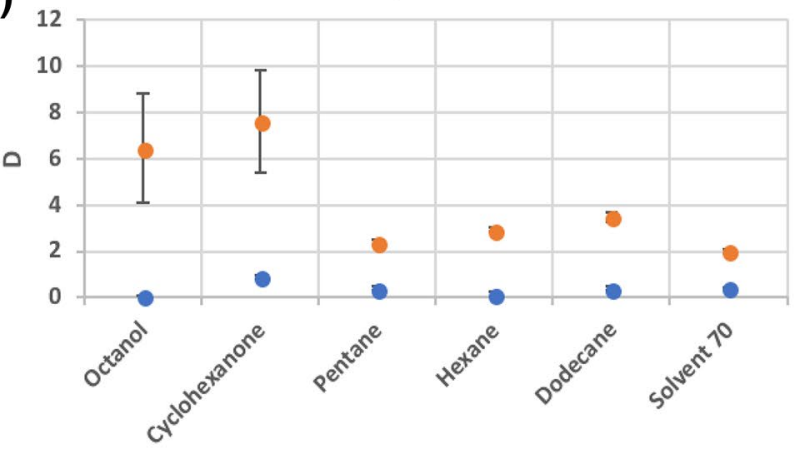

Diluents

(d)

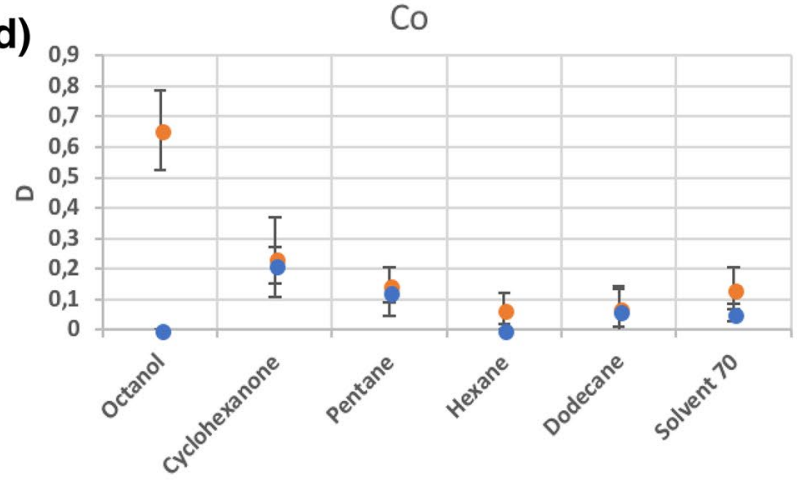

Diluents
- Glycolic

- Maleic

- Glycolic Maleic 
Table 2 The values of the dielectric constant for cyclohexanone, 1-octanol, dodecane, hexane, pentane, and Solvent 70 [28]

\begin{tabular}{lc}
\hline Diluent & $\begin{array}{l}\text { Dielectric } \\
\text { constant }\end{array}$ \\
\hline Cyclohexanone & 18.3 \\
1-Octanol & 10.3 \\
Dodecane & 2.03 \\
Hexane & 1.89 \\
Pentane & 1.84 \\
Solvent 70 & 1.8 \\
\hline
\end{tabular}

Hexane-containing solvent, especially for tests with the maleic leachate, showed no extraction of either Co or B, and very low extraction of $\mathrm{Fe}$, which is very interesting from the selectivity standpoint. Pentane also showed great efficiency, which could be because it is closer in terms of alkyl-chain length to D2EHPA alkyl chains than to big aliphatic alkanes, such as dodecane and Solvent 70. Also, the dielectric constants of these diluents follow the trend of the results (Table 2), as pentane is the most effective and has the lowest dielectric constant after Solvent 70 . The fact that octanol and cyclohexanone diluents tend to allow more transition metal extraction can be explained, as they can participate themselves in an extraction mechanism using the oxygen atoms and bind to the metal ions [28].

Stripping of the REEs from D2EHPA-containing organic phases can be easily performed using acids of various strengths (e.g., $2 \mathrm{M} \mathrm{HCl}$ ) to tailor to the efficiency of stripping as shown in previous research [7, 22].

\section{Conclusions}

This work was aimed at providing both a leaching method and a separation process for the recycling of REEs from magnets using organic acids. The main goal was to minimize the use of mineral acids and optimize experimental conditions in the solvent extraction step to find the most suitable and optimal design for industrial use. Glycolic, maleic, and ascorbic acids had not been used before for the leaching of neodymium magnet waste. It was shown that these could be a feasible replacement for mineral acids in the leaching step.

Leaching was more efficient in glycolic and maleic acids than in ascorbic acid, dissolving the studied elements almost entirely and reaching equilibrium in $400 \mathrm{~min}$. As expected, increasing the concentration of the acids and decreasing the $\mathrm{S} / \mathrm{L}$ ratio enhanced the leaching efficiency for all metals tested, giving results comparable to those where mineral acids were used. Temperature increase was beneficial for the leaching in ascorbic acid, allowing the leaching to reach values above $95 \%$ extraction at $70{ }^{\circ} \mathrm{C}$ for REEs. These results make the organic acids as efficient as inorganic acids at the conditions tested, and these could prove to be a promising leaching alternative.

Extraction was tested using glycolic and maleic leachates. Low distribution ratios and poor separation factors between REEs and other metals were determined for TBP, Cyanex 272, and 923, using Solvent 70 as a diluent. However, for TODGA with the maleic leachate, and for D2EHPA with both leachates, D values showed that REEs were extracted preferentially from the aqueous phase, contrary to $\mathrm{Fe}, \mathrm{Co}$, and $\mathrm{B}$. This shows that these types of leachate have potential future use on a large scale in terms of selectively extracting REEs from other impurities. Nonpolar aliphatic diluents, such as pentane and hexane, showed the best efficiency.

Funding The research leading to these results has received funding from the European Community's Seventh Framework Programme ([FP7/2007-2013]) under Grant No. 607411 (MC-ITN EREAN: European Rare Earth Magnet Recycling Network). This publication reflects only the authors' views, exempting the Community from any liability. Project website: http://www.erean.eu. The authors would like to acknowledge with thanks for the contribution with additional funding toward the ENVIREE project (Environmentally friendly and efficient methods for extraction of rare-earth elements (REE) from secondary sources-ERA-MIN Project 2015-2017).

\section{Compliance with Ethical Standards}

Conflict of interest On behalf of all authors, the corresponding author states that there is no conflict of interest.

Open Access This article is distributed under the terms of the Creative Commons Attribution 4.0 International License (http://creativeco mmons.org/licenses/by/4.0/), which permits unrestricted use, distribution, and reproduction in any medium, provided you give appropriate credit to the original author(s) and the source, provide a link to the Creative Commons license, and indicate if changes were made.

\section{References}

1. Cotton S (2006) Lanthanide and actinide chemistry. Wiley, Chichester

2. Binnemans K, Jones PT, Müller T, Yurramendi L (2018) Rare earths and the balance problem: how to deal with changing markets? J Sustain Metall 4(1):126-146

3. Yang Y, Walton A, Sheridan R, Güth K, Gauß R, Gutfleisch O, Buchert M, Steenari B-M, Van Gerven T, Jones PT, Binnemans K (2017) REE recovery from end-of-life NdFeB permanent magnet scrap: a critical review. J Sustain Metall 3(1):122-149

4. Tunsu C, Petranikova M, Gergorić M, Ekberg C, Retegan T (2015) Reclaiming rare earth elements from end-of-life products: a review of the perspectives for urban mining using hydrometallurgical unit operations. Hydrometallurgy 156:239-258

5. Binnemans K, Jones PT, Blanpain B, Van Gerven T, Yang Y, Walton A, Buchert M (2013) Recycling of rare earths: a critical review. J Clean Prod 51:1-22

6. European Commission (2018) Critical raw materials for the EU. In: European Commission (ed) Report of the ad-hoc working 
group on defining critical raw materials. European Commission, Brussels

7. Xie F, Zhang TA, Dreisinger D, Doyle F (2014) A critical review on solvent extraction of rare earths from aqueous solutions. Miner Eng 56:10-28

8. Lyman JW, Palmer GR (1993) Recycling of neodymium iron boron magnet scrap. United States Department of the Interior, Washington DC

9. Yoon H-S, Kim C-J, Chung KW, Lee S-J, Joe A-R, Shin Y-H, Lee S-I, Yoo S-J, Kim J-G (2014) Leaching kinetics of neodymium in sulfuric acid from E-scrap of $\mathrm{NdFeB}$ permanent magnet. Korean J Chem Eng 31(4):706-711

10. Gergoric M, Ekberg C, Foreman MRSJ, Steenari B-M, Retegan T (2017) Characterization and leaching of neodymium magnet waste and solvent extraction of the rare-earth elements using TODGA. J Sustain Metall 3(3):638-645

11. Önal MAR, Aktan E, Borra CR, Blanpain B, Van Gerven T, Guo M (2017) Recycling of NdFeB magnets using nitration, calcination and water leaching for REE recovery. Hydrometallurgy 167:115-123

12. Lee C, Chen Y, Liao C, Popuri SR, Tsai S, Hung C (2013) Selective leaching process for neodymium recovery from scrap NdFe-B magnet. Metall Mater Trans 44(13):5825-5833

13. Tanaka Y, Zhang Q, Saito F (2002) Sonochemical recovery of metals from recording media. J Chem Eng Jpn 35(2):173-177

14. Itakura $\mathrm{T}$, Sasai R, Itoh $\mathrm{H}$ (2006) Resource recovery from $\mathrm{Nd}-$ $\mathrm{Fe}-\mathrm{B}$ sintered magnet by hydrothermal treatment. J Alloy Compd 408:1382-1385

15. Behera SS, Parhi PK (2016) Leaching kinetics study of neodymium from the scrap magnet using acetic acid. Sep Purif Technol 160:59-66

16. Koivistoinen OM, Kuivanen J, Barth D, Turkia H, Pitkänen JP, Penttilä M, Richard P (2013) Glycolic acid production in the engineered yeasts Saccharomyces cerevisiae and Kluyveromyces lactis. Microb Cell Fact 12:82

17. Sigel A, Operschall BP, Sigel H, Roland KO (2017) Lead: its effects on environment and health. University of Zurich, Zurich, pp 319-402
18. Yang X, Zhang Y, Fang X (2014) Rare earth element recycling from waste nickel-metal hydride batteries. J Hazard Mater 279:384-388

19. Mohammadi M, Forsberg K, Kloo L, Martinez De La Cruz J, Rasmuson $\AA$ (2015) Separation of ND(III), DY(III) and Y(III) by solvent extraction using D2EHPA and EHEHPA. Hydrometallurgy 156:215-224

20. Yang J, Retegan T, Steenari B-M, Ekberg C (2016) Recovery of indium and yttrium from Flat Panel Display waste using solvent extraction. Sep Purif Technol 166:117-124

21. Kuipa PK, Hughes MA (2002) Diluent effect on the solvent extraction rate of copper. Sep Sci Technol 37:1135-1152

22. Gergoric M, Ekberg C, Steenari B-M, Retegan T (2017) Separation of heavy rare-earth elements from light rare-earth elements via solvent extraction from a neodymium magnet leachate and the effects of diluents. J Sustain Metall 3(3):601-610

23. Rydberg J, Cox M, Musicas C, Choppin GR (2004) Solvent extraction principles and practice. Taylor \& Francis, Boca Raton

24. Önal MAR, Jönsson C, Zhou W, Van Gerven T, Guoa M, Walton A, Blanpain B (2017) Comparative oxidation behavior of $\mathrm{Nd}-$ $\mathrm{Fe}-\mathrm{B}$ magnets for potential recycling methods: effect of hydrogenation pre-treatment and magnet composition. J Alloys Compd 728C:727-738

25. Haynes W (ed) CRC handbook of chemistry and physics, 94th edn. CRC Press, Boca Raton

26. do Nascimento ALCS, Teixeira JA, Nunes WDG, Gomes DJC, Gaglieri C, Treu-Filho O, Pivatto M, Caires FJ, Ionashiro M (2017) Thermal behavior of glycolic acid, sodium glycolate and its compounds with some bivalent transition metal ions in the solid state. J Therm Anal Calorim 130:1463

27. Pelendritis M (2017) Nuclear fuel waste extraction: third phase revisited. The University of Manchester, Manchester

28. Wypych G (2008) Knovel solvents-a properties database. ChemTec Publishing, Toronto 